Bond University

Research Repository

\title{
Ability of fitness testing to predict injury risk during initial tactical training: a systematic review and meta-analysis
}

Tomes, Colin D; Sawyer, Sally; Orr, Robin; Schram, Ben

Published in:

Injury Prevention

DOI:

10.1136/injuryprev-2019-043245

Licence:

CC BY-NC

Link to output in Bond University research repository.

Recommended citation(APA):

Tomes, C. D., Sawyer, S., Orr, R., \& Schram, B. (2020). Ability of fitness testing to predict injury risk during initial tactical training: a systematic review and meta-analysis. Injury Prevention, 26(1), 67-81.

https://doi.org/10.1136/injuryprev-2019-043245

\section{General rights}

Copyright and moral rights for the publications made accessible in the public portal are retained by the authors and/or other copyright owners and it is a condition of accessing publications that users recognise and abide by the legal requirements associated with these rights.

For more information, or if you believe that this document breaches copyright, please contact the Bond University research repository coordinator. 
Title:

The ability of fitness testing to predict injury risk during initial tactical training: A systematic review and meta-analysis

\section{Authors:}

Tomes, $C^{1}$., Sawyer, $S^{1}$., \& Schram, $B^{1,2} \&$ Orr, $R^{1,2}$

${ }^{1}$ Faculty of Health Sciences and Medicine, Bond Institute of Health and Science, Bond University, Gold Coast QLD, Australia

${ }^{2}$ Tactical Research Unit, Bond University, Gold Coast, QLD, Australia

Contact: Colin D Tomes, colin.tomes@student.bond.edu.au +61 75595 5828, 14 University Dr. Robina QLD 4226

Word Count: 3993 


\section{ABSTRACT}

Regardless of their role within an organization, all tactical personnel require sufficient physical fitness to be able to complete daily occupational tasks safely and effectively. One challenge faced by tactical training institutions lies in the use of physical training to increase fitness, especially in less fit trainees, when physical training itself is known to contribute to injury risk. Therefore, the aims of this review were to identify studies that have investigated associations between fitness measures and injuries, critically appraise the quality of these studies, and synthesize key findings to inform tactical organizations. Twenty-seven studies were ultimately included. Mean Critical Appraisal Skills Programme score was 10.6/12 (9-12) for cohort studies and 9.5/10 (9-10) for case-control studies. A meta-analysis was performed on publications studying a timed, fixed-distance run, reporting hazard or risk ratios categorically and the number of injuries in each group. The combined risk ratio was determined to be $2.34(95 \% \mathrm{Cl}$ 2.02-2.70). This indicates a significant increase in risk of injury during training for personnel who performed in the slowest quartile or quintile when compared to peers in the fastest quartile or quintile, which follows given this high volume of distance weight bearing activity required by tactical personnel. Muscular endurance tests, such as pushups, sit-ups and pull-ups were less conclusive in their predictive abilities. Functional strength tests were effective predictors, but only four studies reported on a measure of strength, and only two were functional measures, indicating a need for further study in this area. 


\section{KEY MESSAGES}

What is already known

- Relationships exist between a trainee's physical fitness and their risk of suffering an injury during tactical training

\section{What this study adds}

- Quantifies the predictive ability of run time across studies, providing consensus on the topic

- Illustrates the deficiencies in research/application of strength, rather than endurance-based testing, which shows promise, but is limited in its evidence

\section{INTRODUCTION}

Tactical personnel can be defined as those individuals whose sworn duty it is to protect their country and/or community, and who may place themselves at risk for this purpose.(1) Regardless of the specific role within an organization, all tactical personnel require sufficient physical fitness to be able to complete occupational tasks safely and effectively. Common tasks may include carrying loads over distance, responding to disasters, negotiating harsh terrain, subduing human threats, evacuating casualties, and performing heavy physical labour, such as digging, chopping, and lifting.(2, 3) In order to work under these varied and often extreme physical demands, many tactical organizations assess the physical fitness of personnel against departmental standards prior to entry, during training, and at regular intervals during service. $(4,5)$ 
In order to prepare trainees for the rigors of their profession, trainees are frequently subjected to strenuous and regular physical training sessions aimed at increasing their fitness.(6-8) Entering this training environment may represent a sudden change in activity levels for many trainees $(7,9)$ which is a known contributor to injury incidence $(10)$ along with other factors such as previous injury (11) and increased BMI.(12-15) These factors may be compounded by poor physical fitness levels at training commencement, necessitating additional physical training and consequent stress. $(15,16)$ As a result of these factors, initial entry trainees are known to be at an elevated risk of injury beyond that of their operational peers (17) and those who are less fit are less likely to complete training than their fitter counterparts.(18) Thus, a major challenge faced by tactical training institutions lies in the use of physical training to increase fitness, especially in less fit trainees, when physical training itself is known to contribute to injury risk.(19)

A potential approach to mitigate this risk is identification of at risk personnel based on their performance in fitness testing. This approach is supported by research suggesting a strong relationship between low levels of physical fitness and injury risk.(12-14) For example, Pope, et al.(18) found that Australian Army recruits who performed poorly in their initial 20m progressive shuttle run score were more likely to be injured during training. However, the methods and results of this research vary substantially, precluding a definitive understanding of how fitness levels during recruit training may be related to a trainee's risk of injury. For example, while several studies have reported significant association between slow run time and injury risk,(11, 15, 20, 21) Armstrong, et al. found no significant relationship between run time 
and injury in a cohort of US Navy Midshipmen.(22) Given these inconsistencies in predictive ability, the aims of this systematic review were to identify studies that have investigated associations between fitness measures and injuries in tactical trainees, critically appraise the methodological quality of these studies, and synthesize key findings to inform tactical organizations.

\section{METHODS}

\section{Search Method}

Literature databases were systematically searched using combinations of keywords (Table 1), Boolean operators, and database heading terms. The selection of keywords was initially guided by keywords used in a sample of known related articles. $(23,24)$ Next, filters were applied in each database, where available. In databases where these filters were not uniform with PubMed search options, the most similar filter was applied. In order to ensure that studies were applicable to current tactical training contexts and injury reporting procedures, only studies conducted within the last 20 years were included. The Defense Technical Information Center (DTIC) was also searched, as this database includes grey literature not found in traditional academic databases but is still relevant to the tactical research community. Duplicate articles were detected and removed by Endnote Software (Clarivate Analytics, Philadelphia, USA). Additional duplicates not automatically captured were removed manually (Figure 1). (Insert Figure 1 Here) 
Table 1: Keyword searches by database

\begin{tabular}{|c|c|c|c|c|c|c|c|c|}
\hline Database & Filters Applied & Target variable & & Exclusion Terms & & Population & & Outcome \\
\hline PubMed & $\begin{array}{l}\text { Sort By: Best Match, } \\
\text { English Language, } \\
\text { Publication Date } \\
\text { 1998-2018, Human } \\
\text { Subjects, Full text } \\
\text { available }\end{array}$ & $\begin{array}{l}\text { “Exercise Testing" OR } \\
\text { “Physical Fitness" [Mesh] } \\
\text { OR “Task Performance and } \\
\text { Analysis" [Mesh] OR } \\
\text { "Athletic Performance" } \\
\text { [Mesh] OR “Physical } \\
\text { Conditioning" OR "Self- } \\
\text { Assessment" [Mesh] OR } \\
\text { "fitness testing" OR } \\
\text { "periodized training" OR } \\
\text { "physical training" OR } \\
\text { "fitness training" }\end{array}$ & NOT & $\begin{array}{l}\text { "Psychometrics" } \\
\text { [Mesh] } \\
\text { OR“Psychological Tests" } \\
\text { [Mesh] OR "Brain } \\
\text { Injuries" [Mesh] OR } \\
\text { "Amputation, } \\
\text { Traumatic" [Mesh] OR } \\
\text { "Limb loss" }\end{array}$ & AND & $\begin{array}{l}\text { "Emergency } \\
\text { Responders" [Mesh] OR } \\
\text { "Military Personnel" } \\
\text { [Mesh] OR “Police" } \\
\text { [Mesh] OR "Sheriff" OR } \\
\text { “Patrol Officer"OR “Law } \\
\text { Enforcement" OR } \\
\text { "tactical athlete" OR } \\
\text { "police cadet" OR } \\
\text { "trainee" OR "recruit" } \\
\text { OR “candidate" OR “FBI" }\end{array}$ & AND & $\begin{array}{l}\text { "Accidents, } \\
\text { Occupational" [Mesh] } \\
\text { OR "Wounds and } \\
\text { Injuries" [Mesh] OR } \\
\text { "injury prevention" } \\
\text { OR "injury } \\
\text { prediction" }\end{array}$ \\
\hline
\end{tabular}




\begin{tabular}{|c|c|c|c|c|c|c|c|c|}
\hline CINAHL & $\begin{array}{l}\text { Abstract available, } \\
\text { English Language }\end{array}$ & $\begin{array}{l}\text { (MH “Exercise Test") OR } \\
\text { (MH "Task Performance } \\
\text { and Analysis") OR (MH } \\
\text { "Resistance Training") OR } \\
\text { (MH “Physical Fitness") OR } \\
\text { (MH “Athletic } \\
\text { Performance") OR (MH } \\
\text { “Physical Conditioning") } \\
\text { Assessment } \\
\text { Screen } \\
\text { Testing (MH “Self- } \\
\text { Assessment") OR "fitness } \\
\text { testing" OR “periodized } \\
\text { training" OR "physical } \\
\text { training" OR "fitness } \\
\text { training" }\end{array}$ & NOT & $\begin{array}{l}\text { (MH “Psycholometrics”) } \\
\text { OR (MH “Psychological } \\
\text { Tests”) OR (MH Brain } \\
\text { Injuries) OR } \\
\text { "Amputation" OR “Limb } \\
\text { Loss" }\end{array}$ & AND & 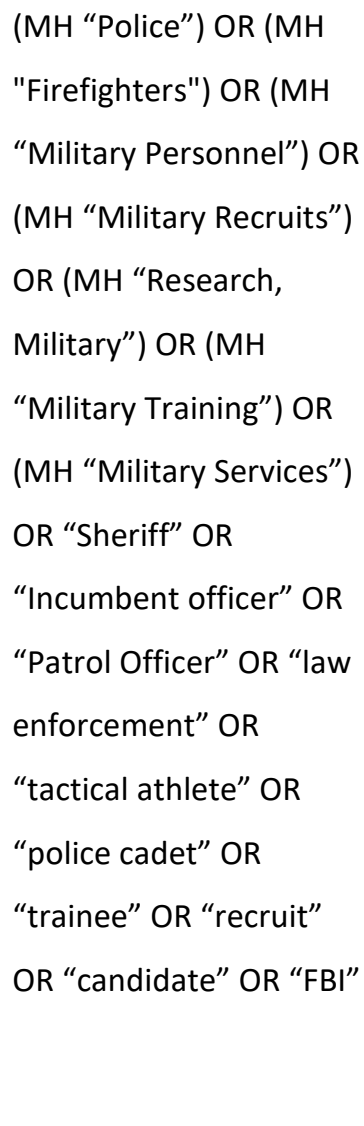 & AND & $\begin{array}{l}\text { (MH "Wounds and } \\
\text { Injuries") OR (MH } \\
\text { "Accidents, } \\
\text { Occupational) OR } \\
\text { "injury prevention" } \\
\text { OR “injury } \\
\text { prediction" }\end{array}$ \\
\hline
\end{tabular}




\begin{tabular}{|c|c|c|c|c|c|c|c|c|}
\hline $\begin{array}{l}\text { SPORT } \\
\text { Discus }\end{array}$ & $\begin{array}{l}\text { 1998-2018, English } \\
\text { Abstract, Journal } \\
\text { Article }\end{array}$ & $\begin{array}{l}\text { "Fitness Testing" OR } \\
\text { "Exercise testing" OR "Task } \\
\text { performance and analysis" } \\
\text { OR "Athletic Performance" } \\
\text { OR "Physical fitness" OR } \\
\text { "fitness screening" OR } \\
\text { “injury screening" OR "self- } \\
\text { assessment" OR "fitness } \\
\text { assessment OR "physical } \\
\text { assessment" OR } \\
\text { "periodized training" OR } \\
\text { "physical training" OR } \\
\text { "fitness training" }\end{array}$ & NOT & $\begin{array}{l}\text { Psychometrics OR } \\
\text { "Psychological Test*" } \\
\text { "Brain injur*” OR } \\
\text { "concussion" OR } \\
\text { "amputation" OR "limb } \\
\text { loss" }\end{array}$ & AND & $\begin{array}{l}\text { Police OR Firefighters OR } \\
\text { Military OR Soldier OR } \\
\text { "Military Recruit" OR } \\
\text { "Emergency Responder" } \\
\text { OR "Law Enforcement" } \\
\text { OR "Sheriff" OR } \\
\text { "Incumbent officer" OR } \\
\text { “Patrol Officer” OR “law } \\
\text { enforcement" OR } \\
\text { "tactical athlete” OR } \\
\text { "police cadet” }\end{array}$ & AND & $\begin{array}{l}\text { Injur* OR Accident } \\
\text { OR Trauma OR } \\
\text { “injury prevention" } \\
\text { OR “injury } \\
\text { prediction" }\end{array}$ \\
\hline Embase & $\begin{array}{l}\text { 1998-2018, English, } \\
\text { Humans, Abstract } \\
\text { available }\end{array}$ & $\begin{array}{l}\text { 'Physical performance'/exp } \\
\text { OR 'athletic } \\
\text { performance'/exp } \\
\text { OR 'fitness'/exp OR } \\
\text { 'occupational health'/exp } \\
\text { OR 'exercise test'/exp OR } \\
\text { 'screening test'/exp OR } \\
\text { "fitness testing” }\end{array}$ & NOT & $\begin{array}{l}\text { 'Psychometry'/exp OR } \\
\text { 'psychologic test'/exp } \\
\text { OR 'neuropsychological } \\
\text { test'/exp OR 'brain } \\
\text { injury'/exp OR } \\
\text { 'concussion'/exp OR } \\
\text { 'amputation'/exp OR } \\
\text { “limb loss” }\end{array}$ & AND & $\begin{array}{l}\text { 'military } \\
\text { phenomena'/exp OR } \\
\text { 'police'/exp OR 'fire } \\
\text { fighter'/exp OR 'law } \\
\text { enforcement'/exp OR } \\
\text { 'rescue personnel'/exp } \\
\text { OR 'army'/exp OR } \\
\text { 'soldier'/exp OR “sheriff” } \\
\text { OR “law enforcement” } \\
\text { OR “tactical athlete” OR }\end{array}$ & AND & $\begin{array}{l}\text { 'occupational } \\
\text { accident'/exp OR } \\
\text { 'accidental } \\
\text { injury'/exp OR } \\
\text { 'avulsion injury'/exp } \\
\text { OR 'concussion'/exp } \\
\text { OR 'musculoskeletal } \\
\text { injury'/exp OR 'head } \\
\text { and neck injury'/exp } \\
\text { OR 'nervous system }\end{array}$ \\
\hline
\end{tabular}

This article has been accepted for publication in Injury Prevention, 2019 following peer review, and the Version of Record can be accessed online at http://dx.doi.org/10.1136/injuryprev-2019-043245 


\begin{tabular}{|c|c|c|c|c|c|c|c|c|}
\hline & & & & & & $\begin{array}{l}\text { “police cadet" OR } \\
\text { "trainee” OR "recruit" } \\
\text { OR “candidate" OR “FBI" }\end{array}$ & & $\begin{array}{l}\text { injury'/exp OR 'pelvis } \\
\text { injury'/exp OR ‘sport } \\
\text { injury'/exp OR 'soft } \\
\text { tissue injury'/exp OR } \\
\text { “injury prevention” } \\
\text { OR “injury } \\
\text { prediction" }\end{array}$ \\
\hline DTIC & $\mathrm{N} / \mathrm{A}$ & $\begin{array}{l}\text { "Fitness testing" OR } \\
\text { "fitness assessment" OR } \\
\text { "exercise testing" OR } \\
\text { "fitness screening" }\end{array}$ & NOT & $\begin{array}{l}\text { Psychometric* OR } \\
\text { "psychological test*" }\end{array}$ & AND & $\begin{array}{l}\text { Police OR Officer OR } \\
\text { Firefighter OR Soldier OR } \\
\text { Recruit OR Trainee OR } \\
\text { Sheriff }\end{array}$ & AND & $\begin{array}{l}\text { injur* OR accident OR } \\
\text { trauma }\end{array}$ \\
\hline
\end{tabular}




\section{Article Screening}

Study eligibility criteria were applied manually through screening of study titles, abstracts and publication metadata to remove articles that were not relevant to the scope of this review (e.g. needlestick injuries, hearing injuries, radiation exposure). Following this process articles were screened against a priori inclusion criteria; these being: a) Study investigated a relationship between one or more direct measures of physical fitness and injury during training, b) study included data on injury or injury risk, c) study followed a tactical population, d) study was peerreviewed, original, primary research, and e) study was published after 1998 . Once all included articles had been gathered, they were compared against emerging exclusion criteria; these being a) reported on a training program or other intervention, b) did not report association between a fitness measure and injury, c) assessed medical provider access, irrespective of reason, d) single event follow-up, e) did not follow subjects through a period of training, or f) investigated heat injury.

\section{Quality Assessment}

Eligible publications identified through the literature search, screening and selection processes were then critically appraised to assess methodological quality using the Critical Appraisal Skills Programme (Middleway, Oxford, UK). Studies were independently assessed by two authors (SS \& $\mathrm{CT}$ ) and measured for agreement by a third author (RO) using Cohen's kappa. The CASP toolkit provides checklists to facilitate accurate and fair appraisal of studies, based on method design. Included studies were suitable for either the CASP cohort study checklist or the CASP case-control study checklist. The cohort study checklist contains 12 questions for study quality 
assessment. The first two questions relate to screening and the following 10 guide reviewers

through the assessment of validity, relevance, methodology and result quality. The case-control checklist comprises 11 questions, the first three of which are focused on screening and the following eight questions assess validity, design effectiveness, power, and applicability.

Questions were scored on a binary scale of either ' 1 ' for questions that can be answered as 'yes' or '0' for those which are answered 'no' or indeterminate. To ensure validity of score reporting, authors completed CASP analysis separately. Any study with a CASP score varying by more than one point between reviewers was re-assessed by a third party. Questions seven through nine on both the Cohort Study CASP and Case-Control Study CASP were condensed into one item, as they are all closely related, and Questions seven and eight cannot be answered dichotomously. Therefore, Cohort papers were then scored out of 12 possible points and CaseControl papers were scored out of 10 possible points. The results for each study are included in Table 2.

\section{Data Extraction}

Full details of data extracted from each article can also be found in Table 2. Author, title, participants, demographics as reported, selected measures of fitness (e.g., aerobic fitness, muscle endurance, and/or muscular strength/power), injury definition, outcome measure, statistical analysis, key results, and CASP score were included. For brevity, only those results meeting statistical significance were included. Data extracted for meta-analysis were also 
included in Table 2. However, all findings reported in studies meeting selection criteria were included, regardless of statistical significance.

\section{Meta-Analysis}

Studies were selected for meta-analysis based on the following criteria: a) study investigated a timed running event and injury risk, b) data were reported categorically, and c) the total number of injuries in each group were obtainable from published data. A random-effects model was used to account for differences between tactical subpopulations, run lengths and sample sizes. Studies were automatically weighted by sample size and confidence interval precision by Revman 5.1 (Cochrane Institute, London, UK). To account for differences in categorization (quartile or quintile), only the fastest and slowest group from each study were included. Final aggregate risk ratio was determined by Mantel-Haenszel analysis of event (injury) incidence difference between fastest and slowest groups. Manual sensitivity analysis showed the study Blacker, et al.(25) accounted for $61 \%$ of overall heterogeneity and therefore was excluded from final analysis. Ultimately, seven studies were included.(10, 11, 15, 21, 26-28)

\section{RESULTS}

The results of search, screening, and selection processes are documented in the PRISMA(29) flow diagram (Figure 1). A total of 1199 publications were captured in the initial search. 135 duplicates were removed automatically, and a further 35 duplicates were removed manually. Of the remaining 1029 articles, 971 did not meet title or abstract relevance based on the initial research question. 58 abstracts were then assessed further based on formal inclusion criteria. 
The final 58 publications were screened by full text against study exclusion criteria ultimately leaving 27 publications for review.

\section{Critical Appraisal}

The mean CASP score was 10.6/12 \pm 0.96 (range 9-12) for the cohort studies and 9.5/10 \pm 0.7 (range 9-10) for the case-control studies. Of the cohort studies, five studies scored 12/12, eight scored 11/12, eight scored 10/12, and four scored 9/12 (See Table 2). Of the case control studies, one scored 10/10 (30), and one study scored 9/10 (22). The level of agreement between the two authors, as measured by Cohen's kappa, $(k=0.750)$ was considered a 'substantial agreement'.(31)

\section{Research Metadata}

\section{Study Design}

Twenty-five of the studies were of a retrospective cohort design with the remaining two studies being of a case-control design.(22, 30) The United States accounted for the largest number of studies, with 15. Three studies were from Finland,(32-34) two studies originated from the United Kingdom, $(20,35)$ two from Israel, $(36,37)$ two from Australia, $(38,39)$ and two from Switzerland. $(40,41)$ One study was from Malta.(42)

Demographics 
11 studies included both male and female participants. Eight articles followed males only,(5, 14, $28,34,35,37,41,43)$ five followed females only, $(13,14,21,27,36)$ and three studies did not report sexes of their participants.(38-40)

Three tactical subpopulations were represented; the largest being military populations, with 24 articles. Two studies followed Australian Police trainees, $(38,39)$ and one study followed US Federal Bureau of Investigation trainees.(10)

Injury Definition

The evaluated injuries and nomenclature varied substantially across studies. Eleven studies included any musculoskeletal injury, while the other publications reported the following injury definitions: Seven studies reported lower limb stress fractures,(14, 21, 22, 27, 28, 32, 37), with one specifically considering femoral neck stress fractures.(30) Two articles recorded any injury, without further description.(10, 16) Two articles considered any musculoskeletal injury requiring hospitalisation, $(13,32)$ two articles considered musculoskeletal injury requiring referral, $(14,20)$ one article was specific to time loss injury,(11) and one article reported any lower limb injury.(42)

\section{Fitness Assessments and Predictive Ability}

Aerobic Fitness

Results of the Meta-Analysis and the forest plot can be found in Figure 2. The aggregate risk ratio was determined to be 2.34 (95\% Cl 2.02-2.70), indicating a significant increase in risk of 
injury during training for personnel who perform in the bottom quartile or quintile when compared to peers in the fastest quartile or quintile. (Insert Figure 2 Here) Of all studies reporting any measure of aerobic fitness, 19 of 21 reported a significant finding. Of the two studies that did not, one study was a smaller, case-control design (22) and the other was investigating femoral neck stress fractures exclusively.(30)

\section{Muscular Fitness}

A total of ten studies $(5,10,11,16,22,30,33,34,44,45)$ investigated relationships between pushups and injury. Four $(5,11,22,33,44)$ reported no significant relationship between timed pushup count and injury risk during training. Of these, one limited their definition of injury to those requiring hospitalization.(32) Three studies considered only lower limb injuries, and yet all reported a significant finding. $(22,30,45)$ All others did not anatomically limit their definitions. Of the studies reporting a significant finding, two found their results were limited to males only $(10,22)$, another found pushups were predictive only of acute injury, and not overuse injury (34) and one was of a smaller, case-control design.(30)

Thirteen studies $(5,10,11,16,22,27,28,30,33,41,43-45)$ investigated a sit-up or crunch test. Of these, eight found no significant relationship between timed sit-up or crunch count and injury risk during training. Two studies favoring sit-up count had very specific definitions of injury; one investigated femoral neck stress fractures (30) and another investigated only injuries resulting in time lost from training.(11) One study found statistically significant predictive ability of the situp test in males, but not females.(16) 
Three studies reported no significant relationship between timed pullup or chin-up count and injury risk during training. $(27,34,43)$ All studies investigating pullups or chin-ups had a broad definition of injury; any acute or overuse musculoskeletal injury was considered. While only one study suggested pullup count was an effective predictor,(20) it did report a significant result for both males and females. One study reporting no relationship between pullup count and injury during training investigated a female only cohort.(27)

\section{Muscular Strength}

Four studies reported on one or more measures of strength.(16, 25, 37, 46) Two of the studies found significant relationships between grip strength(46) and leg press strength(37) and injury risk during training. The studies that did not find a significant result used dynamometer testing rather than functional test methods. $(16,25)$

\section{Muscular Power}

Two studies investigated a measure of muscular power $(25,47)$. One used an external load (dynamic lift strength in a 1 rep max movement) and another used vertical jump height. Both studies reported a statically significant finding. 
Table 2, Data extraction table

\begin{tabular}{|c|c|c|c|c|c|c|}
\hline $\begin{array}{l}\text { Title/ } \\
\text { Authors }\end{array}$ & Participants & Fitness Measures & Injury Reporting & Follow Up & Results $^{\dagger}$ & $\begin{array}{l}\text { CASP } \\
\text { Score }\end{array}$ \\
\hline $\begin{array}{l}\text { Armstrong et al. } 2004 \\
\text { Stress Fracture Injury in } \\
\text { Young Military Men and } \\
\text { Women }\end{array}$ & $\begin{array}{c}\text { US Naval Academy Midshipmen } \\
\text { Cases }(n=31) \\
\text { Age (F): } 18.5 \pm 0.17 \text { years } \\
\text { Age (M): } 18.9 \pm 0.21 \text { years } \\
\text { Height (F): } 163.9 \pm 1.74 \mathrm{~cm} \\
\text { Height (M): } 181.6 \pm 1.97 \mathrm{~cm} \\
\text { Weight (F): } 62.9 \pm 2.31 \mathrm{~kg} \\
\text { Weight (M): } 78.3 \pm 2.01 \mathrm{~kg} \\
\text { BMI (F): } 23.0 \pm 0.63 \\
\text { BMI (M): } 23.8 \pm 0.54 \\
\text { Controls }(n=31) \\
\text { Age (F): } 18.4 \pm 0.17 \text { years } \\
\text { Age (M): } 19.3 \pm 0.21 \text { years } \\
\text { Height (F): } 166.2 \pm 1.74 \mathrm{~cm} \\
\text { Height (M): } 177.2 \pm 1.97 \mathrm{~cm} \\
\text { Weight (F): } 65.0 \pm 2.31 \mathrm{~kg} \\
\text { Weight (M): } 76.8 \pm 2.01 \mathrm{~kg} \\
\text { BMI (F): } 23.5 \pm 0.63 \\
\text { BMI (M): } 24.5 \pm 0.54\end{array}$ & $\begin{array}{l}\text { Initial Strength Test: } \\
1 \text { mile run } \\
\text { Max pushups in } 2 \text { mins } \\
\text { Max Sit ups in } 2 \text { mins }\end{array}$ & $\begin{array}{c}\text { Lower extremity stress } \\
\text { fracture: } \\
\text { Tibial }(\mathrm{n}=43) \\
\text { Metatarsal }(\mathrm{n}=5) \\
\text { Femoral }(\mathrm{n}=3) \\
\text { Fibular }(\mathrm{n}=3) \\
\text { Other }(\mathrm{n}=4)\end{array}$ & - & $\begin{array}{l}\text { Male cases completed } \\
\text { fewer pushups } \\
\text { Cases: } 59 \pm 4 \\
\text { Controls: } 72 \pm 4\end{array}$ & $\begin{array}{c}9 / 10 \\
\text { Case- } \\
\text { Control }\end{array}$ \\
\hline $\begin{array}{l}\text { Kupferer et. al. } \\
\qquad 2014 \\
\text { Femoral neck stress } \\
\text { fracture in Air Force } \\
\text { basic trainees }\end{array}$ & $\begin{array}{l}\text { US Air Force Initial Entry Trainees } \\
\qquad n=47 \text { cases and } 94 \text { controls }\end{array}$ & $\begin{array}{l}\text { US Air Force Fitness Assessment } \\
\text { T1: Max pushups in } 1 \mathrm{~min} \\
\text { T2: Max sit-ups in } 1 \mathrm{~min} \\
\text { T3: } 1.5 \text { mile run for time }\end{array}$ & $\begin{array}{c}\text { Femoral Neck Stress } \\
\text { Fracture } \\
47 \text { cases }\end{array}$ & 8.5 weeks & $\begin{array}{c}\text { T1: controls completed } \\
\text { more reps OR } 0.58(0.38- \\
0.88) \\
\text { T3: cases had slower run } \\
\text { times OR } 1.49(1.19-186) \\
\text { Male findings were } \\
\text { especially significant OR } \\
3.24(1.16-9.04)\end{array}$ & $\begin{array}{c}10 / 10 \\
\text { Case- } \\
\text { control }\end{array}$ \\
\hline $\begin{array}{l}\text { Bedno et al. } 2013 \\
\text { Effect of pre-accession } \\
\text { physical fitness on } \\
\text { training injuries among } \\
\text { US Army recruits }\end{array}$ & 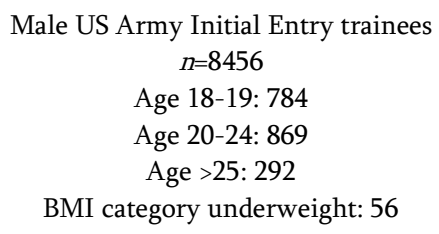 & $\begin{array}{c}\text { Harvard Step Test: } \\
120 \text { steps per minute onto a } 16 \\
\text { inch platform for } 5 \text { minutes }\end{array}$ & $\begin{array}{l}\text { Outpatient presentation } \\
\text { during training for } \\
\text { musculoskeletal pain, a } \\
\text { sprain or strain, } \\
\text { arthropathy, bone stress } \\
\text { injury or tendonopathy }\end{array}$ & 90 days & $\begin{array}{c}\text { HR } 1.31(1.07-1.61) \text { for } \\
\text { test failure }\end{array}$ & $\begin{array}{l}\text { 10/12 } \\
\text { Cohort }\end{array}$ \\
\hline
\end{tabular}

This article has been accepted for publication in Injury Prevention, 2019 following peer review, and the Version of Record can be accessed online at http://dx.doi.org/10.1136/injuryprev-2019-043245 


\begin{tabular}{|c|c|c|c|c|c|c|}
\hline & $\begin{array}{c}\text { BMI category normal: } 791 \\
\text { BMI category overweight: } 722 \\
\text { BMI category obese: } 376\end{array}$ & $\begin{array}{l}\text { Scored as pass/fail; those who } \\
\text { completed all } 5 \text { minutes at pace } \\
\text { passed }\end{array}$ & & & & \\
\hline $\begin{array}{l}\text { Blacker et al. } 2008 \\
\text { Risk Factors for Training } \\
\text { Injuries among British } \\
\text { Army Recruits }\end{array}$ & $\begin{array}{c}\text { British Army Initial Recruit Trainees } \\
n=13417 \\
n=11937 \text { males } \\
n=1480 \text { females } \\
\text { Age: } 20.5 \pm 3.2 \text { years } \\
\text { Height: } 175 \pm 8 \mathrm{~cm} \\
\text { Weight: } 70 \pm 10 \mathrm{kgs} \\
\text { BMI: } 23 \pm 2\end{array}$ & $\begin{array}{c}\text { T1: } 2.4 \mathrm{~km} \text { run } \\
\text { T2: } 20 \mathrm{~m} \text { shuttle runs to failure } \\
\text { T3: Max Chin-ups (heaves) } \\
\text { T4: 4kg ammunition box hold for } \\
\text { 4mins } \\
\text { T5: IKD measurement of back } \\
\text { extension strength } \\
\text { T6: Static lift strength } \\
\text { T7: Dynamic lift strength }\end{array}$ & $\begin{array}{l}\text { Training related acute } \\
\text { or overuse injury } \\
\text { resulting in referral for } \\
\text { remedial instruction }\end{array}$ & 183 days & $\begin{array}{c}\text { T1: } \\
\text { QT1: (fastest) HR 1.00 } \\
\text { QT2: HR 1.66 (1.18-2.34) } \\
\text { QT3: } 2.44(1.76-3.37) \\
\text { QT4: } 3.56(2.61-4.85) \\
\text { QT5: (slowest) 6.25 (4.66- } \\
8.39) \\
\text { T2: } \\
\text { QT1: (fewest repeats) HR } \\
1.00 \\
\text { QT2: HR 0.63 (0.47-0.76) } \\
\text { QT3: HR 0.43 (0.33-0.55) } \\
\text { QT4: HR 0.33 (0.24-0.44) } \\
\text { QT5: (most repeats) HR } \\
\text { 0.20 (0.14-0.29) } \\
\text { T3: } \\
\text { QT1: (fewest reps) HR } \\
1.00 \\
\text { QT2: HR 0.56 (0.46-0.69) } \\
\text { QT3: HR 0.44 (0.35-0.55) } \\
\text { QT4: HR 0.43 (0.35-0.52) } \\
\text { QT5: (most reps) HR 0.30 } \\
\text { (0.24-0.38) } \\
\text { T4: } \\
\text { QT1: (shortest hold) HR } \\
1.00 \\
\text { QT2: HR 0.50 (0.38-0.66) } \\
\text { QT4: HR 0.42 (0.33-0.55) } \\
\text { QT5: HR 0.47 (0.37-0.60) } \\
\text { T5: } \\
\text { QT1: (weakest) HR 1.00 } \\
\text { QT2: HR 0.57 (0.47-0.70) } \\
\text { QT3: HR 0.38 (0.31-0.48) } \\
\text { QT4: HR 0.46 (0.38-0.58) } \\
\text { QT5: HR 0.37 (0.30-0.47) } \\
\text { T6: } \\
\text { QT1: (lowest) HR 1.00 }\end{array}$ & $\begin{array}{c}12 / 12 \\
\text { Cohort }\end{array}$ \\
\hline
\end{tabular}

This article has been accepted for publication in Injury Prevention, 2019 following peer review, and the Version of Record can be accessed online at http://dx.doi.org/10.1136/injuryprev-2019-043245 


\begin{tabular}{|c|c|c|c|c|c|c|}
\hline & & & & & $\begin{array}{c}\text { QT2: HR } 0.61(0.50-0.75) \\
\text { QT3: HR } 0.48(0.39-0.60) \\
\text { QT4: HR 0.48 (0.39-0.60) } \\
\text { QT5: (highest) HR 0.38 } \\
(0.30-0.48) \\
\text { T7: } \\
\text { QT1: (lowest) HR 1.00 } \\
\text { QT2: HR 0.52 (0.42-0.66) } \\
\text { QT3: HR 0.37 (0.28-0.47) } \\
\text { QT4: HR 0.45 (0.38-0.55) } \\
\text { QT5: HR 0.41 (0.33-0.51) }\end{array}$ & \\
\hline $\begin{array}{c}\text { Cowan et al. } 2012 \\
\text { Step Test Performance } \\
\text { and Risk of Stress } \\
\text { Fractures Among Female } \\
\text { Army Trainees }\end{array}$ & $\begin{array}{l}\text { Female US Army initial entry trainees } \\
\qquad n=1568 \\
\text { Age } 18-19: 746 \\
\text { Age 20-24: } 588 \\
\text { Age }>25: 234 \\
\text { BMI category underweight: } 73 \\
\text { BMI category normal: } 1032 \\
\text { BMI category overweight: } 428 \\
\text { BMI category obese: } 35\end{array}$ & $\begin{array}{c}\text { Harvard Step Test: } \\
120 \text { steps per minute onto a } 16 \\
\text { inch platform for } 5 \text { minutes } \\
\text { Scored as pass/fail; those who } \\
\text { completed all } 5 \text { minutes at pace } \\
\text { passed }\end{array}$ & $\begin{array}{c}\text { 64.2\% experienced } \geq 1 \\
\text { injury } \\
7.0 \% \text { stress fractures } \\
4.3 \% \text { equivocal stress } \\
\text { fracture } \\
57.7 \% \text { MSK injury } \\
98 \% \text { of those who had a } \\
\text { stress fracture also had } \\
\text { an MSK injury }\end{array}$ & 180 days & $\begin{array}{l}\text { Stress fracture: IRR } 1.76 \\
\text { (1.18-2.63) } \\
\text { MSK injury: IRR } 1.35 \\
(1.16-1.57)\end{array}$ & $\begin{array}{l}\text { 9/12 } \\
\text { Cohort }\end{array}$ \\
\hline $\begin{array}{l}\text { Grier et. al. } 2011 \\
\text { Risk Factors for Injuries } \\
\text { in the US Army } \\
\text { Ordnance School }\end{array}$ & $\begin{array}{l}\text { US Army Advanced Individual Training } \\
\text { Recruits } \\
n=4255 \\
n=3757 \text { males } \\
n=498 \text { females } \\
\text { Age } 17-19: 2309 \\
\text { Age } 20-24: 1495 \\
\text { Age }>25: 451\end{array}$ & $\begin{array}{l}\text { US Army Physical Fitness Test } \\
\text { T1: } 2 \text { minute max pushups } \\
\text { T2: } 2 \text { minutes max sit-ups } \\
\text { T3: } 2 \text { mile run for time }\end{array}$ & $\begin{array}{l}\text { Time-loss injury: injury } \\
\text { of any type listed by the } \\
\text { medical provider the } \\
\text { trainee presented to } \\
\text { that required limited } \\
\text { duty or restriction to } \\
\text { quarters for one or more } \\
\text { days }\end{array}$ & $\begin{array}{c}10, \\
9, \\
13, \\
16, \text { or } \\
12 \text { weeks, } \\
\text { specific to } \\
\text { each career } \\
\text { field }\end{array}$ & $\begin{array}{c}\text { Male T2: } \\
\text { QR1: (most reps) HR 1.00 } \\
\text { QR2: HR 1.21 (1.01-1.46) } \\
\text { QR3: HR 1.35 (1.12-1.62) } \\
\text { QR4: (fewest reps) HR } \\
\text { 1.23 (1.02.-1.48) } \\
\text { Male T3: } \\
\text { QR1 (fastest): HR 1.00 } \\
\text { QR4 (slowest): HR 1.41 } \\
\text { (1.18-1.69) } \\
\text { Female T3: } \\
\text { QR1 (fastest): HR 1.00 } \\
\text { QR2: HR 1.58 (1.09-2.30) } \\
\text { QR4 (slowest): HR 2.17 } \\
\text { (1.50-3.14) }\end{array}$ & $\begin{array}{c}\text { 9/12 } \\
\text { Cohort }\end{array}$ \\
\hline $\begin{array}{c}\text { Hall } 2017 \\
\text { Relationship between } \\
1.5 \text { mile run time, injury }\end{array}$ & $\begin{array}{c}\text { Male British Army initial entry training } \\
\text { recruits } \\
n=3446\end{array}$ & Timed 1.5-mile run & $\begin{array}{l}591 \text { MSK injuries were } \\
\text { reported; } \\
63 \% \text { were acute } \\
78 \% \text { were lower limb }\end{array}$ & $\begin{array}{l}\text { June 2009- } \\
\text { June } 2011\end{array}$ & $\begin{array}{c}\text { 0-20th percentile (fastest): } \\
\text { RR } 1.00 \\
\text { 21-40th percentile (562- } \\
590 \text { sec): } \\
\text { RR } 1.25(0.96-1.64)\end{array}$ & $\begin{array}{l}\text { 11/12 } \\
\text { Cohort }\end{array}$ \\
\hline
\end{tabular}

This article has been accepted for publication in Injury Prevention, 2019 following peer review, and the Version of Record can be accessed online at http://dx.doi.org/10.1136/injuryprev-2019-043245 


\begin{tabular}{|c|c|c|c|c|c|c|}
\hline $\begin{array}{c}\text { risk and training } \\
\text { outcome in British Army } \\
\text { Recruits }\end{array}$ & & & $\begin{array}{c}42 \% \text { were reported to } \\
\text { be caused by running } \\
71 \% \text { occured in the first } \\
6 \text { weeks }\end{array}$ & & $\begin{array}{l}\text { 41-60th percentile: } \\
\text { RR 1.38 (1.06-1.81) } \\
\text { 61-80th percentile: } \\
\text { RR 1.60 (1.24-2.06) } \\
\text { 81-90th percentile: } \\
\text { RR 1.96 (1.48-2.60) } \\
\text { 91-100th percentile } \\
\text { (slowest): } \\
\text { RR 2.37 (1.82-3.09) }\end{array}$ & \\
\hline $\begin{array}{l}\text { Hoffman et. al. } 1999 \\
\text { The Effect of Leg } \\
\text { Strength on the } \\
\text { Incidence of Lower } \\
\text { Extremity Overuse } \\
\text { injuries during Military } \\
\text { Training }\end{array}$ & 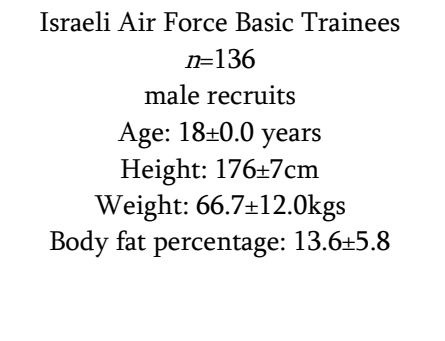 & $\begin{array}{l}\text { T1: } 1 \text { Repetition Max leg press } \\
\text { T2: } 2 \mathrm{~km} \text { run for time }\end{array}$ & $\begin{array}{c}\text { Stress fracture and } \\
\text { related events: } \\
58 \text { clinical visits } \\
\text { suggestive of overuse } \\
\text { injury } \\
32 \text { episodes of limited } \\
\text { duty } \\
12 \text { diagnoses of stress } \\
\text { fracture }\end{array}$ & 9 weeks & $\begin{array}{c}\text { Means were compared to } \\
\text { results 1SD below means } \\
\text { T1: } \\
\text { RR } 4.7(1.7-13.6) \\
1 \text { Repetition Max leg press } \\
\text { per kg of body weight: RR } \\
5.2(1.8-14.7) \\
\text { T2: RR } 1.9 \text { (95\%CI 0.6- } \\
6.6)\end{array}$ & $\begin{array}{c}\text { 9/12 } \\
\text { Cohort }\end{array}$ \\
\hline $\begin{array}{l}\text { Jones et. al. } 2017 \\
\text { Impact of physical } \\
\text { fitness and body } \\
\text { composition on injury } \\
\text { risk among active young } \\
\text { adults: A study of Army } \\
\text { trainees }\end{array}$ & $\begin{array}{l}\text { US Army initial entry trainees } \\
\qquad n=184670 \\
n=143398 \text { males } \\
n=41727 \text { females } \\
\text { Age (M): } 22.6 \pm 4.9 \\
\text { Age (F): } 22.3 \pm 4.8 \\
\text { Height (M): } 176 \pm 6 \mathrm{~cm} \\
\text { Height (F): } 162 \pm 6 \mathrm{~cm} \\
\text { Weight (M): } 77.8 \pm 13.2 \\
\text { Weight (F): } 61.8 \pm 8.6 \\
\text { BMI (M): } 25.1 \pm 3.7 \\
\text { BMI (F): } 23.3 \pm 2.7\end{array}$ & $\begin{array}{l}\text { US Army Physical Fitness Test } \\
\text { T1: } 2 \text { minute max pushups } \\
\text { T2: } 2 \text { minute max sit-ups } \\
\text { T3: } 2 \text { mile run for time }\end{array}$ & $\begin{array}{l}\text { Any acute or overuse } \\
\text { MSK injury reported } \\
\text { during the training } \\
\text { period }\end{array}$ & $\begin{array}{l}\text { All } 10 \\
\text { week } \\
\text { training } \\
\text { periods } \\
\text { from Oct } \\
2009 \text { to } \\
\text { Sept } 2012\end{array}$ & $\begin{array}{c}\text { Male T3: } \\
\text { QT1: 1.0 } \\
\text { QT2: RR 1.3 (1.2-1.3) } \\
\text { QT3: RR 1.5 (1.4-1.5) } \\
\text { QT4: RR 1.8 (1.7-1.8) } \\
\text { QT5: RR 2.5 (2.4-2.5) } \\
\text { Female T3: } \\
\text { QT1: (fastest) RR 1.0 } \\
\text { QT2: RR 1.3 (1.3-1.4) } \\
\text { QT3: RR 1.5 (1.4-1.5) } \\
\text { QT4: RR 1.7 (1.6-1.8) } \\
\text { QT5: (slowest) RR 2.1 } \\
\text { (2.0-2.2) }\end{array}$ & $\begin{array}{c}\text { 10/12 } \\
\text { Cohort }\end{array}$ \\
\hline $\begin{array}{l}\text { Knapik, et. al. } 2011 \\
\text { Injury rates and injury } \\
\text { risk factors among } \\
\text { Federal Bureau of } \\
\text { Investigation new agent } \\
\text { trainees }\end{array}$ & $\begin{array}{c}\text { Federal Bureau of Investigation new } \\
\text { agent trainees } \\
n=531 \\
n=426 \text { males } \\
n=105 \text { females }\end{array}$ & $\begin{array}{l}\text { Physical Fitness Test } \\
\text { T1: sit-ups } \\
\text { T2: push-ups } \\
\text { T3: } 300 \text {-meter sprint } \\
\text { T4: } 1.5 \text { mile run }\end{array}$ & $\begin{array}{l}\text { Any physical damage to } \\
\text { the body requiring } \\
\text { medical care or medical } \\
\text { compensation at one or } \\
\text { more times during } \\
\text { training }\end{array}$ & 1 year & $\begin{array}{c}\text { Male T3: } \\
\text { QR4: HR 1.71 (1.03-2.84) } \\
\text { Female T3: } \\
\text { Tertile 3: HR 2.23 (1.06- } \\
\text { 4.70) } \\
\text { Male T4: } \\
\text { QR4: HR 2.06 (1.30-2.75) } \\
\text { Female T4: }\end{array}$ & $\begin{array}{c}\text { 12/12 } \\
\text { Cohort }\end{array}$ \\
\hline
\end{tabular}

This article has been accepted for publication in Injury Prevention, 2019 following peer review, and the Version of Record can be accessed online at http://dx.doi.org/10.1136/injuryprev-2019-043245 


\begin{tabular}{|c|c|c|c|c|c|c|}
\hline & & & & & $\begin{array}{l}\text { Tertile 3: HR } 1.95 \text { (1.00- } \\
\text { 3.80) }\end{array}$ & \\
\hline $\begin{array}{l}\text { Knapik, et. al. } 2001 \\
\text { Risk factors for training- } \\
\text { related injuries among } \\
\text { men and women in basic } \\
\quad \text { combat training }\end{array}$ & 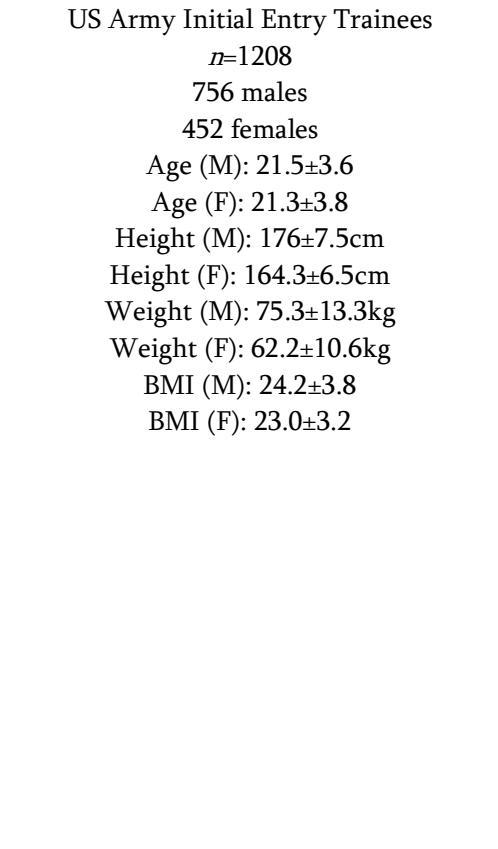 & $\begin{array}{l}\text { US Army Physical Fitness Test } \\
\text { T1: } 2 \text { minute max pushups } \\
\text { T2: } 2 \text { minutes max sit-ups } \\
\text { T3: } 2 \text { mile run for time } \\
\text { Physiological Tests } \\
\text { T4: PeakVO2 treadmill }\end{array}$ & $\begin{array}{l}\text { All Overuse Injuries or } \\
\text { Traumatic Injuries } \\
\text { Time-loss injury } \\
\text { Lower extremity } \\
\text { injuries }\end{array}$ & 8 weeks & $\begin{array}{c}\text { Male T1: } \\
\text { QR3: RR 1.8 (1.2-2.8) } \\
\text { QR4: RR1.8 (1.2-2.8) } \\
\text { Male T2: } \\
\text { QR4: RR 1.6 (1.0-2.4) } \\
\text { Male T3: } \\
\text { QR4: RR 1.6 (1.0-2.4) } \\
\text { Male T4: } \\
\text { Lowest tertile absolute } \\
\text { VO2Peak: RR 2.1(1.0-4.5) } \\
\text { Lowest tertile relative } \\
\text { VO2Peak: RR 1.9(1.0-3.7) } \\
\text { Female T1: } \\
\text { QR2: } 1.6(1.1-2.5) \\
\text { QR3: 1.6(1.1-2.3) } \\
\text { QR4: 1.6(1.1-2.4) } \\
\text { Female T3: } \\
\text { QR3: 1.6 (1.0-2.3) } \\
\text { QR4: 1.9 (1.2-2.8) } \\
\text { Lowest tertile absolute } \\
\text { VO2Peak: RR 1.9 (1.1-3.2) } \\
\text { Lowest tertile relative } \\
\text { VO2Peak: RR 1.9 (1.1-3.4) }\end{array}$ & $\begin{array}{l}10 / 12 \\
\text { Cohort }\end{array}$ \\
\hline $\begin{array}{l}\text { Kodesh et. al. } \\
2015 \\
\text { Examination of the } \\
\text { Effectiveness of } \\
\text { Predictors for } \\
\text { Musculoskeletal Injuries } \\
\text { in Female Soldiers }\end{array}$ & $\begin{array}{l}\text { Female Combat Fitness instructor Course } \\
\text { Trainees (Israel Defense Force) } \\
\qquad n=158 \\
\text { Age: } 18.1-20.2 \text { years (median } 19.0) \\
\text { Height: } 146-181 \mathrm{~cm} \text { (median } 1.64 \text { ) } \\
\text { Weight: } 43-82 \mathrm{~kg} \text { (median } 56) \\
\text { BMI: } 16.1-32.0 \text { (median } 20.8 \text { ) }\end{array}$ & $2 \mathrm{~km}$ run for time & $\begin{array}{c}145 \text { injuries } \\
\text { 80\% lower extremity } \\
\text { MSK } \\
\text { 84\% categorized as } \\
\text { overuse } \\
\text { 37 soldiers lost at least } 2 \\
\text { days of training from } \\
\text { their injury }\end{array}$ & 3 months & $\begin{array}{c}\text { OR: } 1.007 \text { (95\% CI 1.001- } \\
1.014)\end{array}$ & $\begin{array}{l}10 / 12 \\
\text { Cohort }\end{array}$ \\
\hline
\end{tabular}

This article has been accepted for publication in Injury Prevention, 2019 following peer review, and the Version of Record can be accessed online at http://dx.doi.org/10.1136/injuryprev-2019-043245 


\begin{tabular}{|c|c|c|c|c|c|c|}
\hline $\begin{array}{c}\text { Krauss et. al. } \\
\qquad 2017 \\
\text { Excess Stress Fractures, } \\
\text { Musculoskeletal Injuries, } \\
\text { and Health Care } \\
\text { Utilization Among Unfit } \\
\text { and Overweight Female } \\
\text { Army Trainees }\end{array}$ & $\begin{array}{c}\text { Female US Army Initial Entry Trainees } \\
\qquad n=1900 \\
\text { Age 18-19: } 756 \\
\text { Age 20-24: } 596 \\
\text { Age } \geq 25: 233 \\
\text { BMI category underweight: } 76 \\
\text { BMI category normal: } 1039 \\
\text { BMI category overweight: } 432 \\
\text { BMI category obese: } 38\end{array}$ & $\begin{array}{c}\text { Harvard Step Test } \\
120 \text { steps per minute onto a } 16 \\
\text { inch platform for } 5 \text { minutes } \\
\text { Scored as pass/fail; those who } \\
\text { completed all } 5 \text { minutes at pace } \\
\text { passed }\end{array}$ & $\begin{array}{l}\text { MSK injury requiring } \\
\text { healthcare utilization: } \\
\text { Medical encounters } \\
\text { resulting in } \\
\text { musculoskeletal injury } \\
\text { diagnoses and all } \\
\text { physical therapy visits }\end{array}$ & 6 months & $\begin{array}{c}\text { IRR } 1.32 \text { (95\% CI, 1.14- } \\
1.53)\end{array}$ & $\begin{array}{c}11 / 12 \\
\text { Cohort }\end{array}$ \\
\hline $\begin{array}{l}\text { Lisman et. al. } \\
\qquad 2013 \\
\text { Functional movement } \\
\text { screen and aerobic } \\
\text { fitness predict injuries in } \\
\text { military training }\end{array}$ & $\begin{array}{l}\text { Male US Marine Corps Officer Candidate } \\
\text { School Trainees } \\
n=6 \text { wk course: } 447 \text { and } 10 \text { week course: } \\
427 \\
\text { Age: } 22.4 \pm 2.7\end{array}$ & $\begin{array}{c}\text { US Marine Corps Physical Fitness } \\
\text { Test } \\
\text { Pull-ups to exhaustion } \\
\text { Max abdominal crunches in } 2 \\
\text { mins } \\
3 \text { mile run for time }\end{array}$ & $\begin{array}{c}\text { Categorical MSK } \\
\text { injuries } \\
\text { Traumatic or Overuse }\end{array}$ & $\begin{array}{c}6 \text { weeks } \\
10 \text { weeks }\end{array}$ & $\begin{array}{c}\text { T3: } \\
(>20.5 \text { mins to complete }) \\
\text { OR } 1.72 \\
(95 \% \text { CI } 1.29-2.31) \\
(p<0.001)\end{array}$ & $\begin{array}{c}11 / 12 \\
\text { Cohort }\end{array}$ \\
\hline $\begin{array}{l}\text { Mattila, Kuronen, et. al. } \\
\qquad 2007 \\
\text { Nature and risk factors } \\
\text { of injury hospitalization } \\
\text { in young adults: a } \\
\text { follow-up of } 135,987 \\
\text { military conscripts }\end{array}$ & $\begin{array}{l}\text { Finnish Initial Entry Trainees } \\
\qquad n=135987 \\
\text { BMI <25: } 79 \% \text { of trainees } \\
\text { BMI 25-30: } 17 \% \text { of trainees } \\
\text { BMI >30: } 4 \% \text { of trainees } \\
\\
133943 \text { men } \\
2044 \text { women }\end{array}$ & $\begin{array}{c}\text { Max pushups in } 1 \mathrm{~min} \\
\text { Max distance standing long jump } \\
\text { Max sit-ups in } 1 \mathrm{~min} \\
\text { Max back lifts in min } \\
\text { Pull-ups to exhaustion } \\
12 \text { min run for distance (Cooper's } \\
\text { test) }\end{array}$ & $\begin{array}{l}\text { Hospitalization for } \\
\text { lower limb injury } \\
6059 \text { hospitalizations } \\
\text { for injury } \\
\\
2695 \text { (57\%) were joint } \\
\text { sprains/ } \\
\text { dislocations }\end{array}$ & $\begin{array}{c}\text { 6-12 } \\
\text { months }\end{array}$ & $\begin{array}{l}\text { Aggregate fitness score: } \\
\text { Poor: OR 1.0 (referent) } \\
\text { Average: OR 1.0 } \\
\text { (95\% CI 0.9-1.1) } \\
\text { Good: } 1.2 \\
\text { (95\% CI 1.1-1.3) } \\
\text { Excellent: } 1.3 \\
\text { (95\% CI 1.1-1.4) } \\
\text { Aerobic Fitness: } \\
\text { (Coopers test) } \\
\text { (overuse injury only) } \\
\text { Poor: } 1.0 \text { (referent) } \\
\text { Average: } 0.9 \\
\text { (95\% CI 0.8-1.1) } \\
\text { Good: } 1.0 \\
\text { (95\% CI 0.9-1.2) } \\
\text { Excellent: } 1.3 \\
\text { (95\% CI 1.1-1.5) }\end{array}$ & $\begin{array}{l}\text { 9/12 } \\
\text { Cohort }\end{array}$ \\
\hline $\begin{array}{l}\text { Mattila, Niva, et. al. } \\
\qquad 2007 \\
\text { Risk Factors for Bone } \\
\text { Stress Injuries: A }\end{array}$ & $\begin{array}{l}\text { Finnish Initial Entry Trainees } \\
\qquad n=152095 \\
\text { BMI }<20: 17 \% \text { of trainees } \\
\text { BMI 20-25: } 58 \% \text { of trainees } \\
\text { BMI }>25: 25 \% \text { of trainees }\end{array}$ & $\begin{array}{l}\text { Max pushups in } 1 \mathrm{~min} \\
\text { Max distance standing long jump } \\
\text { Max sit-ups in } 1 \mathrm{~min} \\
\text { Max back lifts in min } \\
\text { Pull-ups to exhaustion }\end{array}$ & $\begin{array}{c}\text { Bone stress injuries of } \\
\text { the pelvis, hip, thigh, or } \\
\text { knee } \\
319 \text { cases }\end{array}$ & $\begin{array}{c}\text { 6-12 } \\
\text { months }\end{array}$ & $\begin{array}{l}\text { Aerobic Fitness: } \\
\text { (Coopers test) } \\
\text { QR1: } 1 \text { (referent) } \\
\text { QR2: } 1.0(0.7-1.6) \\
\text { QR3: } 1.3(0.9-2.0)\end{array}$ & $\begin{array}{l}\text { 9/12 } \\
\text { Cohort }\end{array}$ \\
\hline
\end{tabular}

This article has been accepted for publication in Injury Prevention, 2019 following peer review, and the Version of Record can be accessed online at http://dx.doi.org/10.1136/injuryprev-2019-043245 


\begin{tabular}{|c|c|c|c|c|c|c|}
\hline $\begin{array}{l}\text { Follow-up Study of } \\
\text { 102,515 Person-Years }\end{array}$ & $\begin{array}{l}17-29 \text { years } \\
\text { (median 20) } \\
149750 \text { men } \\
2345 \text { women }\end{array}$ & $\begin{array}{c}12 \text { min run for distance } \\
\text { (Cooper's test) }\end{array}$ & & & $\begin{array}{l}\text { Q4: } 1.3(0.8-2.0) \\
\text { Aggregate Strength score: } \\
\text { QR1: } 1 \text { (referent) } \\
\text { QR2: } 0.8(0.5-1.4) \\
\text { QR3: } 1.2(0.8-1.9) \\
\text { QR4: } 1.4(0.7-1.9)\end{array}$ & \\
\hline $\begin{array}{c}\text { Nye et. al. } \\
2016 \\
\\
\text { Description and Rate of } \\
\text { Musculoskeletal Injuries } \\
\text { in Air Force Basic } \\
\text { Military Trainees, 2012- } \\
2014\end{array}$ & $\begin{array}{l}\text { US Air Force basic military trainees } \\
\qquad n=67525\end{array}$ & $\begin{array}{l}\text { PFT (Physical fitness test) } \\
\text { Push up count } \\
\text { Sit up count } \\
1.5 \text { mile run time }\end{array}$ & $\begin{array}{c}12.5 \% \text { injured with } 1 \text { or } \\
\text { more injuries } \mathrm{n}=8448 \\
78.4 \% \mathrm{n}=9147 \text { involved } \\
\text { the lower extremity }\end{array}$ & 8.5 weeks & $\begin{array}{l}\text { Push-up count } \mathrm{p}=<0.001 \\
\text { Sit-up count } \mathrm{p}=<0.001 \\
1.5 \text { mile run } \mathrm{p}=<0.001\end{array}$ & $\begin{array}{l}11 / 12 \\
\text { Cohort }\end{array}$ \\
\hline $\begin{array}{l}\text { Orr, et. al. } \\
2016 \\
\\
\text { Leg Power As an } \\
\text { Indicator of Risk of } \\
\text { Injury or Illness in } \\
\text { Police Recruits }\end{array}$ & $\begin{array}{l}\text { Australian Police recruits } \\
\qquad n=1021\end{array}$ & Vertical jump height & $\begin{array}{c}\text { Injuries alone } 15 \%(\mathrm{n}= \\
158)\end{array}$ & 12 weeks & $\begin{array}{c}\text { Correlation between } \\
\text { Vertical Jump height and } \\
\text { injury and illness } \\
\text { prevalence were } \mathrm{p}=0.003 \\
\text { and } \mathrm{p}=0.001 \text { respectively }\end{array}$ & $\begin{array}{l}\text { 10/12 } \\
\text { Cohort }\end{array}$ \\
\hline $\begin{array}{c}\text { Orr, et. al. } 2017 \\
\text { Grip Strength and Its } \\
\text { Relationship to Police } \\
\text { Recruit Task } \\
\text { Performance and Injury } \\
\text { Risk: A Retrospective } \\
\text { Cohort Study }\end{array}$ & $\begin{array}{l}\text { Police recruits undergoing full-time } \\
\text { training at a police college in Australia } \\
\qquad n=169 \\
\text { No demographic information was } \\
\text { available }\end{array}$ & $\begin{array}{l}\text { Grip strength measured using a } \\
\text { handgrip dynamometer. } \\
\text { Maximum grip strength of right } \\
\text { and left hands was measured to } \\
\text { the nearest kilogram. }\end{array}$ & $\begin{array}{l}\text { An injury was reported } \\
\text { in } 25.4 \% \text { ( }=43 \text { ) of } \\
\text { recruits } \\
\text { Injury data was } \\
\text { collected during the } \\
\text { training period. This } \\
\text { was done from the } \\
\text { reporting protocol by } \\
\text { using a standard } \\
\text { accident and incident } \\
\text { form } \\
\text { No information was } \\
\text { provided regarding } \\
\text { injury type, site or } \\
\text { severity }\end{array}$ & 12 weeks & $\begin{array}{c}\text { Mean grip Left }(\mathrm{kg}) \\
\text { No injury } 42.80 \pm 8.23 \\
\text { Injury } 39.28 \pm 8.92 \\
\mathrm{r}=-0.181(\mathrm{p}=0.018)\end{array}$ & $\begin{array}{l}10 / 12 \\
\text { Cohort }\end{array}$ \\
\hline
\end{tabular}

This article has been accepted for publication in Injury Prevention, 2019 following peer review, and the Version of Record can be accessed online at http://dx.doi.org/10.1136/injuryprev-2019-043245 


\begin{tabular}{|c|c|c|c|c|c|c|}
\hline $\begin{array}{l}\text { Psaila et. al. } \\
2017 \\
\text { Risk factors for lower } \\
\text { leg, ankle and foot } \\
\text { injuries during basic } \\
\text { military training in the } \\
\text { Maltese Armed Forces }\end{array}$ & $\begin{array}{c}\text { Maltese Armed Services Initial Entry } \\
\text { Trainees } \\
n=127 \\
114 \text { males } \\
13 \text { females } \\
\text { Mean age: } 21.7(2.4) \\
\text { Mean BMI: } 24.8\end{array}$ & $\begin{array}{l}\text { Physical Fitness Test: } \\
\text { Max push-ups in } 2 \text { minutes } \\
\text { Max sit-ups in } 2 \text { minutes } \\
1 \text { mile run for time }\end{array}$ & $\begin{array}{l}\text { Lower limb injuries } \\
\text { Most common injuries } \\
\text { per body part were: } \\
\text { medial tibial stress } \\
\text { syndrome in the leg, } \\
\text { acute lateral ankle } \\
\text { sprain in the ankle and } \\
\text { heel fat pad contusion } \\
\text { in the foot } \\
45 \text { injuries (incidence: } \\
0.204 \text { injuries/100 days) }\end{array}$ & 135 days & $\begin{array}{l}\text { Pre-Basic Military } \\
\text { Training fitness test scores } \\
\text { were associated with } \\
\text { injury risk during BMT } \\
\text { (Mann-Whitney } \mathrm{U}= \\
1226, \mathrm{p}=0.039 \text { ) }\end{array}$ & $\begin{array}{l}11 / 12 \\
\text { Cohort }\end{array}$ \\
\hline $\begin{array}{l}\text { Rauh et. al. } \\
\qquad 2006 \\
\text { Epidemiology of stress } \\
\text { fracture and lower- } \\
\text { extremity overuse injury } \\
\text { in female recruits }\end{array}$ & $\begin{array}{c}\text { Female US Marine Corps Initial Entry } \\
\text { Trainees } \\
\qquad n=824 \\
\text { Age } 17-19: 738 \\
\text { Age } \geq 20: 86 \\
\text { BMI category underweight: } 63 \\
\text { BMI category normal: } 737 \\
\text { BMI category overweight: } 20\end{array}$ & $\begin{array}{c}\text { US Marine Corps Physical Fitness } \\
\text { Test } \\
\text { Pull-ups to exhaustion } \\
\text { Max abdominal crunches in } 2 \\
\text { mins } \\
3 \text { mile run for time }\end{array}$ & $\begin{array}{l}\text { Lower-extremity stress } \\
\text { fracture } \\
\text { Non-stress fracture } \\
\text { overuse injuries } \\
399 \text { cases }\end{array}$ & 13 weeks & $\begin{array}{c}\text { Run time, Stress Fracture } \\
\text { QR1 1.0 } \\
\text { QR2 1.4 }(0.5,3.9) \\
\text { QR3 1.1 }(0.4,3.2) \\
\text { QR4 3.3 }(1.4,8.1)\end{array}$ & $\begin{array}{c}11 / 12 \\
\text { Cohort }\end{array}$ \\
\hline $\begin{array}{l}\text { Sefton et. al. } 2016 \\
\text { Prediction of Injuries } \\
\text { and Injury Types in } \\
\text { Army Basic Training, } \\
\text { Infantry, Armor, and } \\
\text { Cavalry Trainees Using a } \\
\text { Common Fitness Screen }\end{array}$ & $\begin{array}{l}\text { US Army Initial Entry Trainees } \\
\qquad \begin{array}{l}n=1788 \\
\text { Age: } 20.48 \pm 3.4\end{array}\end{array}$ & $\begin{array}{l}1-1-1 \text { Test } \\
\text { Max pushups in } 1 \mathrm{~min} \\
\text { Max sit-ups in } 1 \mathrm{~min} \\
1 \text { mile run for time }\end{array}$ & $\begin{array}{c}\text { Acute or overuse MSK } \\
\text { injury } \\
308 \text { traumatic injuries } \\
222 \text { overuse injuries }\end{array}$ & 9-16 weeks & $\begin{array}{c}\text { Ln Run Time: } \\
\text { Estimate: } 1.972(p<0.001)\end{array}$ & $\begin{array}{l}12 / 12 \\
\text { Cohort }\end{array}$ \\
\hline $\begin{array}{l}\text { Shaffer et. al. } \\
1999 \\
\text { Use of simple measures } \\
\text { of physical activity to } \\
\text { predict stress fractures in } \\
\text { young men undergoing a } \\
\text { rigorous physical } \\
\text { training program }\end{array}$ & $\begin{array}{c}\text { US Marine Corps Initial Entry Trainees } \\
\qquad \begin{array}{c}n \text { Phase } 1: 1286 \text { and Phase } 21078 \\
\text { Phase } 1 \text { Age: } 18.9 \pm 2.3 \\
\text { Phase } 2 \text { Age: } \\
18.4 \pm 1.4\end{array}\end{array}$ & $\begin{array}{l}\text { Aerobic Fitness Assessment } \\
1.5 \text { mile run for time }\end{array}$ & $\begin{array}{c}\text { Lower extremity stress } \\
\text { fractures } \\
52 \text { cases } \\
56 \text { total injuries } \\
\text { tibia }(46.4 \%) \\
\text { foot }(39.3 \%) \\
\text { fibula }(5.3 \% \text { t) } \\
\text { heel }(5.3 \%) \\
\text { femur }(1.8 \%) \\
\text { pelvis }(1.8 \%)\end{array}$ & $\begin{array}{c}12 \\
\text { weeks }\end{array}$ & $\begin{array}{l}\text { Initial Strength Test by } \\
1.5 \text { miles run time } \\
\text { QR1: } 1.0 \text { (referent) } \\
\text { QR2: } 0.87(0.27-2.82) \\
\text { QR3: } 1.72(0.65-4.6) \\
\text { QR4: } 3.11(1.26-7.66)\end{array}$ & $\begin{array}{l}10 / 12 \\
\text { Cohort }\end{array}$ \\
\hline
\end{tabular}

This article has been accepted for publication in Injury Prevention, 2019 following peer review, and the Version of Record can be accessed online at http://dx.doi.org/10.1136/injuryprev-2019-043245 


\begin{tabular}{|c|c|c|c|c|c|c|}
\hline $\begin{array}{l}\text { Shaffer et. al. } \\
\qquad 2006 \\
\text { Predictors of stress } \\
\text { fracture susceptibility in } \\
\text { young female recruits }\end{array}$ & $\begin{array}{l}\text { Female Marine Corps Recruits } \\
\qquad n=2962 \\
\text { Aged } 17 \text { to } 33 \text { years } \\
\text { Weight: } 58.17 \mathrm{~kg} \\
\text { BMI: } 21.67\end{array}$ & $\begin{array}{c}\text { Aerobic Fitness Testing } \\
0.75 \text { or } 1 \text { mile run, depending on } \\
\text { recruiting station }\end{array}$ & $\begin{array}{c}\text { Lower Extremity stress } \\
\text { Fractures } \\
152 \text { cases }(5.1 \%) \\
181 \text { total injuries } \\
\text { tibia }(24.9 \%) \\
\text { metatarsals }(22.1 \%) \\
\text { pelvis }(21.6 \%) \\
\text { femur }(19.9 \%)\end{array}$ & $\begin{array}{c}12 \\
\text { weeks }\end{array}$ & $\begin{array}{c}\text { Run Time } \\
\text { Overall Stress Fracture } \\
\text { AOR }(95 \% \text { CI) } \\
\text { QR1: } 1.00 \\
\text { QR2: } 1.21(0.5-2.1) \\
\text { QR3: } 3.41(1.9-6.1) \\
\text { QR4: } 3.54(2.0-6.3)\end{array}$ & $\begin{array}{c}\text { 11/12 } \\
\text { Cohort }\end{array}$ \\
\hline $\begin{array}{c}\text { Taanila et. al. } \\
2015 \\
\\
\text { Risk factors of acute and } \\
\text { overuse musculoskeletal } \\
\text { injuries among young } \\
\text { conscripts: A } \\
\text { population-based cohort } \\
\text { study }\end{array}$ & $\begin{array}{c}\text { Finnish Army Initial Entry Trainees } \\
n=1411 \\
\text { Median Age: } 19 \\
\text { Median BMI: } 22.6 \text { (signals) } \\
23.3 \text { (mortars) } \\
23.4 \text { (anti-tank) } \\
23.6 \text { (engineering) }\end{array}$ & $\begin{array}{c}\text { T1: Max pushups in } 1 \mathrm{~min} \\
\text { T2: Max distance standing long } \\
\text { jump } \\
\text { T3: Max sit-ups in } 1 \mathrm{~min} \\
\text { T4: Max back lifts in min } \\
\text { T5: Pull-ups to exhaustion } \\
\text { T6: } 12 \text { min run for distance } \\
\text { Coopers Test } \\
\text { T7: Muscle Fitness index (sum of } \\
\text { T1-5) } \\
\text { T8: Physical Fitness Index } \\
\text { (T6+100xT7/200) } \\
\\
\text { Graded in categories: poor, fair } \\
\text { good, good, and excellent }\end{array}$ & $\begin{array}{c}\begin{array}{c}\text { Acute or overuse MSK } \\
\text { injury }\end{array} \\
\begin{array}{c}550 \text { acute injuries } \\
(\mathrm{n}=27 \%)\end{array} \\
\begin{array}{c}1351 \text { overuse injuries ( } \\
=51 \%)\end{array} \\
1411 \text { cases }\end{array}$ & 180 days & $\begin{array}{c}\text { Acute Injury } \\
\text { T1: Poor }(<22) \text { HR1.4 (1.1- } \\
1.9) \\
\text { T2: Poor }(<2.00 \mathrm{~m}) \text { HR1.4 } \\
(1.0-2.0) \\
\text { T7: Good (9-12 points) } \\
\text { HR1.5 }(1.0-2.2) \\
\text { Poor }(0-4 \text { points }) \\
\text { HR1.6 }(1.1-2.4) \\
\text { Overuse injury } \\
\text { T1: Poor }(<22) \text { HR }=1.5 \\
(1.2-1.8) \\
\text { T2: Poor }(<2.00 \mathrm{~m}) \text { HR }= \\
1.6(1.2-2.0) \\
\text { T4: Fair good }(>40) \text { HR }= \\
1.3(1.1-1.5) \\
\text { Poor }(<40) \text { HR }=1.9(1.5- \\
2.4) \\
\text { T6: Fair good }(>2200 \mathrm{~m}) \\
\text { HR = 1. } 3(1.1-1.5) \\
\text { Poor }(<2200 \mathrm{~m}) \\
\text { HR = 1.8 }(1.5-2.2) \\
\text { T7: Fair good }(5-8 \text { points }) \\
\text { HR = 1.5 }(1.1-1.9) \\
\text { Poor }(0-4 \text { points }) \text { HR = 1.7 } \\
(1.3-2.3) \\
\text { T8: Fair good }(13.00- \\
\text { 16.99) HR = 1.3 }(1.1-1.5) \\
\text { Poor }(<13.00) \text { HR }=1.8 \\
(1.5-2.2)\end{array}$ & $\begin{array}{c}\text { 11/12 } \\
\text { Cohort }\end{array}$ \\
\hline
\end{tabular}




\begin{tabular}{|c|c|c|c|c|c|c|}
\hline $\begin{array}{l}\text { Wunderlin et. al. } \\
\qquad 2015 \\
\\
\text { Trunk muscle strength } \\
\text { tests to predict injuries, } \\
\text { attrition and military } \\
\text { ability in soldiers }\end{array}$ & $\begin{array}{l}\text { Swiss Army fusilier company recruits } \\
\qquad(\mathrm{n}=230) \\
\text { Age: } 20.4 \pm 1.2 \\
\text { height: } 177.8 \pm 6.4 \mathrm{~cm} \\
\text { Weight: } 73.7 \pm 11.8 \\
\text { BMI: } 23.3 \pm 3.2\end{array}$ & $\begin{array}{c}\text { T1: Max sit-ups in } 2 \text { mins } \\
\text { T2: TMS } \\
\text { Front plank position with } \\
\text { alternating leg elevations at a } 1 \mathrm{~Hz} \\
\text { rhythm }\end{array}$ & $\begin{array}{c}\text { Acute or overuse MSK } \\
\text { injury } \\
111 \text { Acute injuries } \\
81 \text { Overuse injuries } \\
192 \text { total } \\
126 \text { cases }\end{array}$ & 13 weeks & $\begin{array}{c}\text { T2: } \\
\text { Total injury: } 0.58 \text { total } \\
\text { AUC ( } p=0.033 \text { ROC } \\
\text { analysis }) \\
\text { Acute injury: } 0.58 \text { total } \\
\text { AUC ( } p=0.035 \text { ROC } \\
\text { analysis })\end{array}$ & $\begin{array}{c}11 / 12 \\
\text { Cohort }\end{array}$ \\
\hline $\begin{array}{l}\text { Wyss et. al. } \\
\qquad 2012 \\
\\
\text { The Swiss Army physical } \\
\text { fitness test battery } \\
\text { predicts risk of overuse } \\
\text { injuries among recruits }\end{array}$ & $\begin{array}{c}4 \text { Groups of Swiss Army Career-Specific } \\
\text { Initial Entry Trainees } \\
n=459 \\
\text { Rescue Technicians } \\
n=131 \\
\text { Armoured Infantry } \\
n=145 \\
\text { Fusilier Infantry } \\
n=107 \\
\text { Reconnaissance Infantry } \\
n=76\end{array}$ & $\begin{array}{l}\text { T1: Standing Long jump } \\
\text { T2: Seated Shot Put } \\
\text { T3: Trunk Muscle Strength Test } \\
\text { T4: One-leg Standing Test } \\
\text { T5: Progressive Endurance Run }\end{array}$ & $\begin{array}{l}234 \text { total injuries } \\
64.5 \% \text { Overuse origin }\end{array}$ & $\begin{array}{c}18 \\
\text { weeks }\end{array}$ & $\begin{array}{c}\text { Rescue Technician: } \\
\text { T3: } 2.53(1.04-6.15) \\
p=0.025 \\
\text { T4: } 2.24(1.22-4.12) \\
p=0.011 \\
\text { Armoured Infantry: } \\
\text { T3: } 1.63(1.04-2.56) \\
p=0.028 \\
\text { T4: } 2.20(1.39-3.49) \\
p<0.0001 \\
\text { Fusilier Infantry: } \\
\text { T1: } 1.81 \text { (1.12-2.92) } \\
p=0.013 \\
\text { T3: } 2.19(1.31-3.66) \\
p=0.001 \\
\text { T4: } 2.44(1.44-4.14) \\
p<0.0001 \\
\text { T5: } 1.81(1.12-2.92) \\
\text { Reconnaissance Infantry: } \\
\text { T3: } 2.06(1.25-3.40) \\
p=0.005\end{array}$ & $\begin{array}{c}10 / 12 \\
\text { Cohort }\end{array}$ \\
\hline $\begin{array}{l}\text { † All Confidence interval } \\
\text { Legend: } \\
\text { US: United States } \\
\text { T1,2,3,n: Test 1,2,3,n } \\
\text { OR: Odds Ratio } \\
\text { BMI: Body Mass Index } \\
\text { HR: Hazard Ratio } \\
\text { IKD: Isokinetic Dynamon } \\
\text { QT: Quintile } \\
\text { MSK: Musculoskeletal } \\
\text { RR: Risk Ratio }\end{array}$ & reported are at the $95 \%$ level unless otherw & se noted & & & & \\
\hline
\end{tabular}


CI: Confidence Interval

QR: Quartile

IRR: Incidence Rate Ratio

AOR: Adjusted Odds Ratio

This article has been accepted for publication in Injury Prevention, 2019 following peer review, and the Version of Record can be accessed online at http://dx.doi.org/10.1136/injuryprev-2019-043245 


\section{DISCUSSION}

The aim of this study was to identify, appraise and synthesize the findings of studies that investigated relationships between initial tactical training fitness assessments and injuries sustained during that training. Twenty-seven studies were included and were generally of high quality due to the observational method of study designs. While some fitness tests were more conclusive than others in predictive ability effectiveness, our results indicate a more fit tactical trainee is less likely to experience injury during initial training.

While aerobic fitness testing methods were highly variable across studies, cross-study agreement was greatest among this test type. Run distances reported were as low as $1 \mathrm{~km},(21)$ or 12 minutes in duration, $(33,34,44)$ indicating that greater distance tests, such as the US Marine Corps training's three mile test, are not necessarily better predictors of fitness. Therefore, assessors could consider measuring trainees over shorter distances, reducing total training load and thereby reducing injury risk (48). There may also be benefit from time-to-exhaustion testing, which can account for variations in motivation while still reducing overall training volume.(49) It should also be noted that extensive previous research in operational units has indicated regular load carriage activity is essential in acclimating individuals to tolerate load carriage for extended periods of time,(50) and yet no studies have investigated a load carriage test in their battery for initial trainees. Weight bearing activity of all degrees of load and over varying distances is inescapable in both training and operational tactical contexts, $(48,51)$ and the overwhelming percentage of lower-limb and overuse injuries in tactical personnel is reflective of this reality. $(13,22,30,40,51)$ Because timed distance run events rely on aerobic capacity in weight bearing over a distance, this certainly contributes to the definitiveness of their predictive ability.

This article has been accepted for publication in Injury Prevention, 2019 following peer review, and the Version of Record can be accessed online at http://dx.doi.org/10.1136/injuryprev-2019-043245 
Muscular fitness tests (pushups, sit-ups, pull-ups and chin-ups) are less clear in their predictive ability; ten studies investigated a pushup test, $(5,10,11,16,22,30,33,34,44,45)$ with an almost even split. Six identified effective predictive ability, $(10,16,22,30,34,45)$ and four did not $(5,11,22,33,44)$. It is worth noting that three of the studies reporting a significant finding $(22,30,45)$ investigated lower limb injuries in military trainees using similar protocols, indicating pushup tests of this design, in this population, may be a more global measure of fitness and not simply a strict measure of upper body muscular capacity. The most robust of those three studies(45) reported a mean pushup count of 13.5 \pm 8.6 for injured female, $33.4 \pm 12.7$ for injured males, $15.7 \pm 8.9$ for uninjured females and $36.6 \pm 12.3$ for uninjured males.

While eight of the 12 studies considering a sit-up test did not report a significant finding, one of the four studies favouring a sit-up test had very large sample size $(n=67525) .(45)$ There were no clear consistencies between those studies that found a significant relationship between sit-ups or crunches and injury and those that did not. Both military and police populations are represented in the studies finding a significant conclusion. Protocols for sit-up and crunch tests varied widely however, possibly obscuring the effectiveness of the general intent to target gross trunk muscle capability. Drawing a definitive conclusion on the effectiveness of pullup or chin-up tests is limited due to the small number of studies including these tests in their investigations.

Functional strength tests were effective and reported large differences in risk between highest and lowest performers. $(37,46)$ However, there is limited data from which a definitive conclusion can be drawn, especially given that isokinetic dynamometer testing was less effective in predicting injuries.(16, 25) It may be possible that isometric (grip strength) isotonic (leg press) strength are more sensitive to 
performance or injury than isokinetic strength. Further study should focus on the design, implementation and effectiveness of field expedient measures of functional strength.

Muscular Power tests were effective predictors as well in both a military (25) and a law enforcement context.(47) Although both tests (a vertical jump and dynamic 1 rep max lift) reached statistical significance, these results are too limited to draw a definitive conclusion, and further research in this area is necessary.

While it has been suggested that changes to the variables and methods used to predict injury must change in order for injury screening tools to be effective,(52) the ultimate value of fitness testing in the tactical environment is in the assessment's specificity to the operational or training context it is evaluating. The results of our meta-analysis confirm this notion; because military personnel are often required to perform running, marching and load carriage activities on a regular basis, a test that taxes aerobic capacity and the ability to bear weight over time appears to be sufficient in predicting injury without additional modelling.

Differences in approaches to fitness testing should also be noted. In this review the intent of fitness testing was not to predict occupational performance, but to determine whether trainees were more likely to sustain an injury when training itself was the occupational requirement. As such, once training commences, the approach to fitness testing can, and should differ. The intent of training for tactical organizations is to ensure that training prepares personnel for operational duty in real-world scenarios. On this basis, conditioning should focus on operational requirements rather than training for a test which, while predictive of survivability of training at the onset, may not best facilitate the training intent (operational readiness). An example of how this malalignment in physical training occurs was 
highlighted by Orr, et al. (53) where an ankle injury was caused by excessive pushups; a training cadet had performed excessive pushups the night before an obstacle course drill, in order to improve pushup ability for their assessment, and was subsequently too fatigued to properly lower from a 12-foot wall, landing badly and ultimately causing their ankle injury. This phenomenon may be further highlighted in the predictive ability of run times to predict a wide variety of both acute and overuse injuries, not just those of the lower limb. It is likely that those trainees with faster run times experience less metabolic stress during a given training event and are thereby less reliant on their passive structures to accommodate forces experienced during training, and so ultimately are less likely to become injured.

\section{Study Populations}

Military initial entry trainees represented the largest subpopulation of tactical personnel in the studies selected for this review and substantial differences existed between their characteristics; national origin, branch of service and subsequent job tasks, and average age and education level serve as examples.

Some, or all, of these factors could have an effect on injury type, incidence, severity and recovery, which may confound our findings. In some of the studies included in this review, $(38,39)$ organizations required additional privacy measures for study participants, limiting which data could be released publicly in the study manuscripts, further obstructing cross-study comparison. Differences between trainee populations and operational personnel should also be noted; previous research has indicated that personnel undergoing training are more susceptible to injury than their operational peers, $(7,9,54)$ and the job tasks required of different professions not only between tactical fields (emergency responders vs. military vs. firefighting) but within a given profession (such as different military assignments)(11, 40) can also affect the nature of injuries sustained. Perhaps more importantly, the end goals of operational and training personnel are fundamentally different; during training the objective is to complete training 
and operational personnel are required to complete operational tasks. Therefore, the findings of this review may not generalize beyond training populations.

\section{Fitness Testing}

Tactical fitness assessments are often required to be field expedient and completed with a minimum of personnel, training and resources, potentially under adverse conditions and must also be cost and time effective, even when large numbers of personnel are evaluated.(55) Given these demands, most fitness tests in this review consisted of easily implemented indirect measures of muscular fitness and metabolic capacity; body-weight exercises (pushups, sit-ups, pull-ups) and timed distance runs, ranging anywhere from 1.1-4.8km. However, these indirect means of assessment were not always consistent in their predicative ability; it is likely that different classifications or qualities of injury may be more or less sensitive to prediction by the above common fitness measures, and differences in test administration (e.g., definition of technique during muscular fitness assessments) may also play a role in the inconsistency of study outcomes. However, the necessary requirement for military personnel, the primary population of this review, to engage in weight bearing activity across distances on a regular basis likely contributes to the substantial agreement in studies investigating a timed, fixed distance running event, $(48,51)$ and the reasoning behind the selection of endurance, rather than strength or power tests. Even within studies that report similar definitions of injury, common injury classification systems are now undergoing scrutiny for validity and accuracy, as well as improvement in communication, as a number of injury classification systems do not account for various clinically relevant features of soft tissue injury.(56) Improvements in this domain, and further research within the tactical community that applies improved injury classification systems may lead to more consistent outcomes in injury prediction studies using fitness tests.

This article has been accepted for publication in Injury Prevention, 2019 following peer review, and the Version of Record can be accessed online at http://dx.doi.org/10.1136/injuryprev-2019-043245 
Finally, the populations included studies followed are known for underreporting their injuries, especially when the reporting mechanism is not point of care. $(57,58)$ Pope, et al. $(59)$ identified this phenomenon in a comparison of reporting systems, concluding that between 80 and $90 \%$ of all injuries sustained by Australian Army personnel that may require healthcare intervention are not reported to a collection database when compared to a 'point of care' injury reporting system often used in research.

\section{CONCLUSION}

Based on the data from this review, fitness testing results may be a reliable means of identifying individuals undergoing tactical training who are at a higher risk of sustaining an injury during their training. However, significant variation in fitness testing methods between organizations, roles, mission and size of each organization, differences in injury definition between publications and differing statistical approaches limits cross-study comparison and conclusiveness of findings. Nonetheless, all tactical organizations require both trainees and operational personnel to perform weight bearing activity with and without load over varying distances and speed. $(48,51)$ Because such activity is central and universal in many tactical settings, specific focus on weight bearing and loaded activity over distance should be considered and trainees should be appropriately acclimated. Our meta-analysis findings confirm this, with studies reporting categorical, fixed-distance timed run events indicating unequivocally that poor metabolic fitness carries an elevated risk of injury during initial tactical training. Strength and power measures correlated well with injury risk, but only four studies investigated a measure of strength, only two investigated a measure of power and only two of the strength tests were functional measures. Therefore, tactical organizations should promote metabolic fitness of trainees, acclimate trainees to weight bearing over distance, use fitness test results to identify personnel as at elevated risk of injury, and intervene appropriately. Further research should investigate implementation and effectiveness of field expedient measures of functional strength and power. 
Acknowledgments, Competing Interests, Funding and all other required statements

The authors report no conflicts of interest or granted funding for this research.

This article has been accepted for publication in Injury Prevention, 2019 following peer review, and the Version of Record can be accessed online at http://dx.doi.org/10.1136/injuryprev-2019-043245 


\section{References}

1. Joseph A, Wiley A, Orr R, Schram B, Dawes J. The impact of load carriage on measures of power and agility in tactical occupations: A critical review. International journal of environmental research and public health. 2018;15(1):88.

2. MacDonald D, Pope R, Orr R. Differences in physical characteristics and performance measures of part-time and full-time tactical personnel: A critical narrative review. Journal of Military and Veterans Health. 2016;24(1):45.

3. Anderson GS, Plecas D, Segger T. Police officer physical ability testing-Re-validating a selection criterion. Policing: An International Journal of Police Strategies \& Management. 2001;24(1):8-31.

4. Payne W, Harvey J. A framework for the design and development of physical employment tests and standards. Ergonomics. 2010;53(7):858-71.

5. Sefton JM, Lohse KR, McAdam JS. Prediction of Injuries and Injury Types in Army Basic Training, Infantry, Armor, and Cavalry Trainees Using a Common Fitness Screen. Journal of athletic training. 2016;51(11):849-57.

6. Cocke C, Dawes J, Orr RM. The Use of 2 Conditioning Programs and the Fitness Characteristics of Police Academy Cadets. Journal of Athletic Training (Allen Press). 2016;51(11):887-96.

7. Orr RM, Pope R. Optimizing the Physical Training of Military Trainees. Strength \& Conditioning Journal (Lippincott Williams \& Wilkins). 2015;37(4):53-9.

8. Orr RM, Ford K, Stierli M. Implementation of an Ability-Based Training Program in Police Force Recruits. Journal of Strength \& Conditioning Research (Lippincott Williams \& Wilkins). 2016;30(10):2781-7.

9. Orr R, Knapik JJ, Pope R. Avoiding Program-Induced Cumulative Overload (PICO). Journal of special operations medicine : a peer reviewed journal for SOF medical professionals. 2016;16(2):91-5.

10. Knapik JJ, Grier T, Spiess A, Swedler DI, Hauret KG, Graham B, et al. Injury rates and injury risk factors among Federal Bureau of Investigation new agent trainees. BMC public health. 2011;11:920.

11. Grier TL, Morrison S, Knapik JJ, Canham-Chervak M, Jones BH, Grier TL, et al. Risk factors for injuries in the U.S. Army Ordnance School. Military Medicine. 2011;176(11):1292-9.

12. Bedno SA, Cowan DN, Urban N, Niebuhr DW. Effect of pre-accession physical fitness on training injuries among US Army recruits. Work (Reading, Mass). 2013;44(4):509-15.

13. Krauss MR, Garvin NU, Cowan DN, Boivin MR. Excess Stress Fractures, Musculoskeletal Injuries, and Health Care Utilization Among Unfit and Overweight Female Army Trainees. American Journal of Sports Medicine. 2017;45(2):311-6.

14. Cowan DN, Bedno SA, Urban N, Lee DS, Niebuhr DW. Step test performance and risk of stress fractures among female army trainees. American journal of preventive medicine. 2012;42(6):620-4.

15. Jones BH, Hauret KG, Dye SK, Hauschild VD, Rossi SP, Richardson MD, et al. Impact of physical fitness and body composition on injury risk among active young adults: A study of Army trainees. Journal of science and medicine in sport. 2017;20 Suppl 4:S17-s22.

This article has been accepted for publication in Injury Prevention, 2019 following peer review, and the Version of Record can be accessed online at http://dx.doi.org/10.1136/injuryprev-2019-043245 
16. Knapik JJ, Sharp MA, Canham-Chervak M, Hauret K, Patton JF, Jones BH. Risk factors for training-related injuries among men and women in basic combat training. Medicine and science in sports and exercise. 2001;33(6):946-54.

17. Kupferer KR, Bush DM, Cornell JE, Lawrence VA, Alexander JL, Ramos RG, et al. Femoral neck stress fracture in Air Force basic trainees. Military medicine. 2014;179(1):56-61.

18. Pope RP, Herbert R, Kirwan JD, Graham BJ, Pope RP, Herbert R, et al. Predicting attrition in basic military training. Military Medicine. 1999;164(10):710-4.

19. Jones BH, Hauschild VD. Physical Training, Fitness, and Injuries: Lessons Learned From Military Studies. Journal of strength and conditioning research. 2015;29 Suppl 11:S57-64.

20. Blacker SD, Wilkinson DM, Bilzon JLJ, Rayson MP, Blacker SD, Wilkinson DM, et al. Risk factors for training injuries among British Army recruits. Military Medicine. 2008;173(3):278-86.

21. Shaffer RA, Rauh MJ, Brodine SK, Trone DW, Macera CA. Predictors of stress fracture susceptibility in young female recruits. The American journal of sports medicine. 2006;34(1):108-15.

22. Armstrong DW, 3rd, Rue JP, Wilckens JH, Frassica FJ. Stress fracture injury in young military men and women. Bone. 2004;35(3):806-16.

23. Baumgartner N. Physical tests and standards from selection through training to operations. Journal of Science and Medicine in Sport. 2017;20:S8.

24. Tomes C, Orr RM, Pope R. The impact of body armor on physical performance of law enforcement personnel: a systematic review. Annals of Occupational and Environmental Medicine. 2017;29(1):14.

25. Blacker SD, Wilkinson DM, Bilzon JLJ, Rayson MP, Blacker SD, Wilkinson DM, et al. Risk Factors for Training Injuries Among British Army recruits. Military medicine. 2008;173(3):27886.

26. Hall LJ. Relationship between 1.5-mile run time, injury risk and training outcome in British Army recruits. Journal of the Royal Army Medical Corps. 2017;163(6):376-82.

27. Rauh MJ, Macera CA, Trone DW, Shaffer RA, Brodine SK. Epidemiology of stress fracture and lower-extremity overuse injury in female recruits. Medicine and science in sports and exercise. 2006;38(9):1571-7.

28. Shaffer RA, Brodine SK, Almeida SA, Williams KM, Ronaghy S. Use of simple measures of physical activity to predict stress fractures in young men undergoing a rigorous physical training program. American journal of epidemiology. 1999;149(3):236-42.

29. Moher D, Liberati A, Tetzlaff J, Altman DG. Preferred reporting items for systematic reviews and meta-analyses: The PRISMA statement. International Journal of Surgery. 2010;8(5):336-41.

30. Kupferer KR. Femoral neck stress fracture in Air Force basic trainees. Military medicine. 2014;179(1):56-61.

31. Viera AJ, Garrett JM. Understanding interobserver agreement: the kappa statistic. Family medicine. 2005;37(5):360.

32. Mattila VM, Kuronen $\mathrm{P}$, Pihlajamaki $\mathrm{H}$. Nature and risk factors of injury hospitalization in young adults: a follow-up of 135,987 military conscripts. Scand J Public Health. 2007;35(4):41823.

This article has been accepted for publication in Injury Prevention, 2019 following peer review, and the Version of Record can be accessed online at http://dx.doi.org/10.1136/injuryprev-2019-043245 
33. Mattila VM, Niva M, Kiuru M, Pihlajamaki H. Risk Factors for Bone Stress Injuries: A Follow-up Study of 102,515 Person-Years. Medicine \& Science in Sports \& Exercise. 2007;39(7):1061-6.

34. Taanila H, Suni JH, Kannus $P$, Pihlajamaki H, Ruohola JP, Viskari J, et al. Risk factors of acute and overuse musculoskeletal injuries among young conscripts: a population-based cohort study. BMC musculoskeletal disorders. 2015;16:104.

35. Hall LJ. Relationship between 1.5-mile run time, injury risk and training outcome in British Army recruits. Journal of the Royal Army Medical Corps. 2017;163(6):376-82.

36. Kodesh E, Shargal E, Kislev-Cohen R, Funk S, Dorfman L, Samuelly G, et al. Examination of the Effectiveness of Predictors for Musculoskeletal Injuries in Female Soldiers. Journal of Sports Science \& Medicine. 2015;14(3):515-21.

37. Hoffman JR, Chapnik L, Shamis A, Givon U, Davidson B. The effect of leg strength on the incidence of lower extremity overuse injuries during military training. Military Medicine. 1999;164(2):153-6.

38. Orr R, Pope R, Peterson S, Hinton B, Stierli M. Leg Power As an Indicator of Risk of Injury or Illness in Police Recruits. International journal of environmental research and public health. 2016;13(2):237.

39. Orr R, Pope R, Stierli M, Hinton B. Grip strength and its relationship to police recruit task performance and injury risk: A retrospective cohort study. International Journal of Environmental Research and Public Health. 2017;14(8).

40. Wyss T, Von Vigier RO, Frey F, Mader U. The Swiss Army physical fitness test battery predicts risk of overuse injuries among recruits. The Journal of sports medicine and physical fitness. 2012;52(5):513-21.

41. Wunderlin S, Roos L, Roth R, Faude O, Frey F, Wyss T. Trunk muscle strength tests to predict injuries, attrition and military ability in soldiers. The Journal of sports medicine and physical fitness. 2015;55(5):535-43.

42. Psaila $\mathrm{M}$, Ranson $\mathrm{C}$. Risk factors for lower leg, ankle and foot injuries during basic military training in the Maltese Armed Forces. Physical therapy in sport : official journal of the Association of Chartered Physiotherapists in Sports Medicine. 2017;24:7-12.

43. Lisman P, O'Connor FG, Deuster PA, Knapik JJ. Functional movement screen and aerobic fitness predict injuries in military training. Medicine and science in sports and exercise. 2013;45(4):636-43.

44. Mattila VM, Kuronen P, Pihlajamäki H. Nature and risk factors of injury hospitalization in young adults: a follow-up of 135,987 military conscripts. Scandinavian Journal of Public Health. 2007;35(4):418-23.

45. Nye NS, Pawlak MT, Webber BJ, Tchandja JN, Milner MR. Description and Rate of Musculoskeletal Injuries in Air Force Basic Military Trainees, 2012-2014. Journal of Athletic Training (Allen Press). 2016;51(11):858-65.

46. Orr R, Pope R, Stierli M, Hinton B. Grip Strength and Its Relationship to Police Recruit Task Performance and Injury Risk: A Retrospective Cohort Study. International journal of environmental research and public health. 2017;14(8).

47. Orr R, Pope R, Peterson S, Hinton B, Stierli M. Leg Power As an Indicator of Risk of Injury or Illness in Police Recruits. International journal of environmental research and public health. 2016;13(2):237.

This article has been accepted for publication in Injury Prevention, 2019 following peer review, and the Version of Record can be accessed online at http://dx.doi.org/10.1136/injuryprev-2019-043245 
48. Trank TV, Ryman Dh Fau - Minagawa RY, Minagawa Ry Fau - Trone DW, Trone Dw Fau Shaffer RA, Shaffer RA. Running mileage, movement mileage, and fitness in male U.S. Navy recruits. (0195-9131 (Print)).

49. Fukuda DH, Smith AE, Kendall KL, Cramer JT, Stout JR. An Alternative Approach to the Army Physical Fitness Test Two-Mile Run Using Critical Velocity and Isoperformance Curves. Military Medicine. 2012;177(2):145-51.

50. Knapik JJ, Harman EA, Steelman RA, Graham BS. A systematic review of the effects of physical training on load carriage performance. Journal of strength and conditioning research. 2012;26(2):585-97.

51. Orr RM, Johnston V, Coyle J, Pope R. Reported load carriage injuries of the Australian army soldier. Journal of occupational rehabilitation. 2015;25(2):316-22.

52. Newman P. Predicting injuries: Are we using the appropriate statistical approach? Journal of Science and Medicine in Sport. 2017;20:S130-S1.

53. Orr R. The Royal Military College of Duntroon. Physical Conditioning Optimisation Review-Project Report Canberra: ACT: Department of Defence. 2010.

54. Schram B, Pope R, Orr R. Injuries in Australian Army full-time and part-time personnel undertaking basic training. BMC Musculoskeletal Disorders. 2019;20(1).

55. Knapik JJ, Rieger W, Palkoska F, Van Camp S, Darakjy S. United States Army physical readiness training: rationale and evaluation of the physical training doctrine. Journal of strength and conditioning research. 2009;23(4):1353-62.

56. Pollock N, James SLJ, Lee JC, Chakraverty R. British athletics muscle injury classification: a new grading system. British Journal of Sports Medicine. 2014;48:1347-51.

57. Schram B, Orr R, Rigby T, Pope R. An Analysis of Reported Dangerous Incidents, Exposures, and Near Misses amongst Army Soldiers. International journal of environmental research and public health. 2018;15(8).

58. Schram B, Orr R, Pope R. A Profile of Knee Injuries Suffered by Australian Army Reserve Soldiers. International Journal of Environmental Research and Public Health. 2019;16(1). 59. Pope R, Orr R. Incidence rates for work health and safety incidents and injuries in Australian army reserve vs full time soldiers, and a comparison of reporting systems. Journal of Military and Veterans Health. 2017;25(2):16-25. 